\title{
Anatomical variations of cystic duct insertion and their relationship with choledocholithiasis: an MRCP study
}

\author{
Nesrin Gündüz ${ }^{1 *} \mathbb{P}$, Mahmut Bilal Doğan², Mine Alacagöz², Merve Yağbasan', \\ Umut Perçem Orhan Söylemez ${ }^{2}$ and Başak Atalay
}

\begin{abstract}
Background: The frequency of insertion variations of cystic duct (CD) is inconsistent between studies caused to some extent by the way they give the relative frequency of the variations. Moreover, certain insertion variations have been reported to be associated with choledocholithiasis. This study aimed to assess the frequency of CD insertion variations with a comprehensive way of classification in an unselected population in whom MRCP was performed. Moreover, the relationship between the types of variant insertions and choledocholithiasis using MRCP was also assessed. Patients undergoing magnetic resonance cholangiopancreatography (MRCP) were reviewed retrospectively by two radiologists who were blinded to the clinical data. The normal insertion was defined as the union through middle one third of the lateral border of the extrahepatic bile duct. The transverse site (lateral, medial, anterior, and posterior) and the craniocaudal level (high, mid and low) of insertions and their intersections were assessed using axial and coronal slices, respectively. In addition, the frequencies of the CD insertion variations were compared between choledocholithiasis and control (no-choledocholithiasis) groups.

Results: A total of 307 patients ( 124 with choledocholithiasis and 183 controls) were analyzed. A true variant insertion was found in $149(48.5 \%)$ cases. The insertion variations were less frequent in the choledocholithiasis group [50 $(40.3 \%)$ vs. $99(54.1 \%)$, respectively, $p=0.018]$. The frequencies of craniocaudal level of insertion differed significantly between groups ( $p=0.014$ ) that was driven by a lower rate of low medial insertion ( $1.6 \%$ vs. $9.8 \%$, respectively) in the choledocholithiasis group. The frequencies of transverse site of insertion were similar between groups $(p=0.314)$. The low medial insertion was $80.7 \%$ less likely associated with choledocholithiasis even after adjustment for age (Odds ratio: $0.193,95 \%$ Confidence interval: $0.039-0.954, p=0.044)$. The interreader agreement for insertion assessment was good (Cohen's Kappa: 0.748, $p<0.001)$.

Conclusions: The prevalence of CD insertion variations in an unselected population undergoing MRCP is quite high and a mid-posterior insertion is the most common variant type. Insertion variations of $C D$, the low medial insertion in particular, are less common in patients with choledocholithiasis than controls.
\end{abstract}

Keywords: MRCP, Anatomical variation, Choledocholithiasis, Cystic duct insertion

*Correspondence: gunduz.nesrin@gmail.com

${ }^{1}$ Department of Radiology, Göztepe Pof. Dr. Süleyman Yalçın City

Hospital, Faculty of Medicine, İstanbul Medeniyet University, Eğitim M, Dr.

Erkin C. No: 161/1, 34722 Kadıköy, İstanbul, Turkey

Full list of author information is available at the end of the article

\section{Background}

The so-called normal mid-lateral insertion of cystic duct (CD) to extrahepatic bile duct is seen in only $51-72 \%$ of population $[1,2]$. The frequency of true insertion variations is inconsistent between studies caused to some extent by the way they give the relative frequency of 
the variations [2-5]. The variations in terms of origin, course, and insertion may overlap, but they are commonly reported separately which results in ambiguity of variation rates. For instance, parallel course is commonly reported in the frequency tables, but it is unclear whether these cases have either a normal or variant insertion [2]. Yet, parallel coursing, aberrant, and accessory cystic ducts are not true insertion variations, because they do not necessarily involve the union of $\mathrm{CD}$ with extrahepatic bile duct. The short cystic duct is also commonly reported in the distribution of variations table without mentioning the insertion pattern of these ducts [3]. Moreover, some studies provide the low and low-medial insertions separately, although the latter is a subgroup of the former [2]. Other studies have reported only the frequency of low-medial insertion with no information about low insertion $[4,5]$. A CD inevitably should insert at a craniocaudal (high/mid/low) level through a transverse (anterior/posterior/lateral/medial) aspect of the extrahepatic bile duct. However, the craniocaudal insertion level was not provided for cases with anterior or posterior insertions in one study [4]. Hence, the frequencies of the insertion variation types of $\mathrm{CD}$ remain ambiguous. A standardization in the way of reporting the frequencies of $C D$ insertion variations would be plausible.

An increased incidence of common bile duct (CBD) stone formation and recurrence in those with low insertion of cystic duct was proposed [6,7]. Biliary tract stones were seen in one-quarter of cases with posterior and anterior insertions in a recent study [5]. The relationship between $\mathrm{CD}$ insertion variations and choledocholithiasis requires addressing.

There has been a shift from endoscopic retrograde cholangiopancreatography and intraoperative cholangiography to preoperative magnetic resonance cholangiopancreatography (MRCP) for the assessment of biliary anatomy [8]. The MRCP is noninvasive and its diagnostic accuracy to detect gallstones, choledocholithiasis and visualize the anatomy of biliary tree preoperatively is comparable to endoscopic retrograde cholangiopancreatography or intraoperative cholangiography [9].

This study aimed to assess the frequency of CD insertion variations with a comprehensive way of classification in an unselected population in whom MRCP was performed. Moreover, the relationship between the types of variant insertions and choledocholithiasis using MRCP was also assessed.

\section{Methods}

\section{Study population}

This single center retrospective study comprised patients who underwent MRCP in our tertiary referral center between July 2019 and July 2020. Overall 379 (150 with choledocholithiasis and 229 without choledocholithiasis) subjects who were imaged with MRCP were identified from the institutional database. The MRI images were reloaded from the Picture Archiving Communication System of our Institute. Patients whose cystic duct insertion to common hepatic duct could not be clearly identified were excluded (26 from choledocholithiasis group and 46 from control group). The remaining 307 eligible subjects (124 choledocholithiasis and 183 controls) constituted the final study population. The inclusion criteria for choledocholithiasis group were adequate MRCP allowing for the clear assessment of $C D$ insertion and visible gallstone in common bile duct. The inclusion criteria for the control group were absence of choledocholithiasis [Normal MRCP findings $(n=161)$, non-biliary pancreatitis $(n=10)$, malignant biliary strictures $(n=2)$, periampullary masses $(n=5)$, biliary injuries $(n=3)$, pancreatic divisum $(n=2)$ ]. The only exclusion criteria for both groups was inadequate MRCP that was not allowing for thorough assessment of CD insertion [72 (19\%) of 379 reviewed patients]. Institutional Ethics Committee approval was obtained. Patients' age, gender, a previous history of and the elapsed time since cholecystectomy, presence and the type of cystic duct variations were recorded into a database.

\section{MRCP scanning and interpretation}

The patients were scanned using the 1.5 Tesla GE Optima MR450w (General Electric, Chicago, Illinois, USA) system. The MRCP protocol used in our Institute is shown in Table 1.

Two radiologists experienced in abdominal crosssectional imaging (10 and 3 years, respectively) who were blinded to the clinical data reviewed all of the MRCP images on separate sessions for interrater agreement analysis. An additional session was performed

Table 1 MRCP protocol and sequences used for the current study

\begin{tabular}{llll}
\hline & T2W SS-FSE & T2W SS-FSE & 3D MRCP \\
\hline Plane & Axial & Coronal & Coronal \\
Fat suppression & + and - & - & + \\
Flip Angle $\left(^{\circ}\right)$ & 160 & 90 & 90 \\
Slice thickness $(\mathrm{mm})$ & 4 & 4 & 1.4 \\
FOV & 40 & 40 & 36 \\
Matrix $(\mathrm{mm} \times \mathrm{mm})$ & $320 \times 320$ & $320 \times 320$ & $512 \times 224$ \\
TR/TE $(\mathrm{ms})$ & $4000 / 84$ & $3500 / 100$ & $3000 / 720$ \\
Scan time $(\mathrm{ms})$ & 59 & 59 & 176 \\
\hline
\end{tabular}

T2WT2 weighted, SS-FSE single-shot fast spin echo, MRCP magnetic resonance cholangiopancreatography, FOV field of view, TR/TE repetition time and echo time 


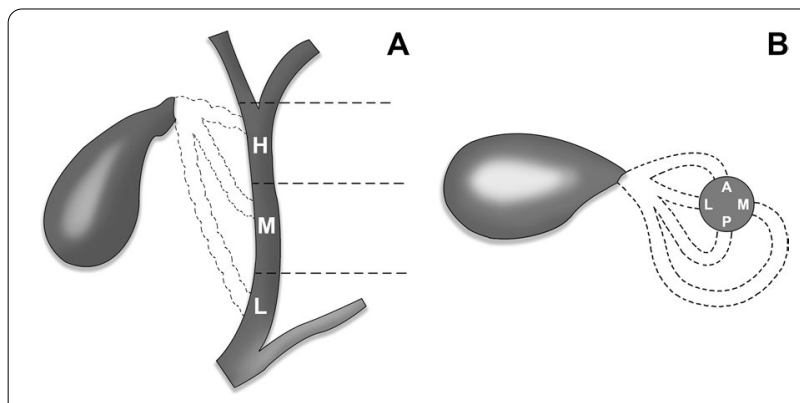

Fig. 1 Schematic demonstration of cystic duct confluence with extrahepatic bile duct. The craniocaudal insertion level is labeled by the letters $\mathrm{H}, \mathrm{M}$ and $\mathrm{L}$ indicating high, mid and low insertions, respectively (a). The transverse site of insertion is labeled by the letters L, A, M and P indicating lateral, anterior, medial and posterior insertions, respectively (b)

for eliminating the discrepancies between readers by consensus.

The CD insertions were classified in terms of craniocaudal level and transverse site of the union of cystic duct with extrahepatic duct (Fig. 1). The craniocaudal level of union was subclassified as high, middle, and low. The whole length of the extrahepatic bile duct was divided into three thirds subjectively. The beginning of this length was accepted as the union of the right and left hepatic ducts where they form the common hepatic duct. The ending point of this length was accepted as the opening of common bile duct into the ampulla of Vater. The transverse site of the union was subclassified as lateral, medial, anterior, and posterior. The transverse site of union of the CD was assessed according to clock method. The lateral union corresponded to 7-11 o'clock, the anterior union corresponded to 11-1 o'clock, the medial union corresponded to 1-5 o'clock, and posterior union corresponded to 5-7 o'clock.

The normal insertion of $\mathrm{CD}$ was defined as the union through middle one third of lateral border. All other insertions were recorded as variant.

\section{Statistical analysis}

Statistical analysis was performed with SPSS 19.0. The normality of continuous variables was analyzed by Shapiro-Wilk test. Descriptive statistics were reported as median with quartiles (25th-75th percentile) for continuous variables since the data distributions were not normal. Categorical variables were reported as frequencies with percentages. Independent two-group comparisons for continuous variables were tested using Mann-Whitney U test. The proportions were compared between the groups using Pearson's Chi-square test in case the assumptions were met. Otherwise, Fisher's exact test was used. Interobserver agreement was also assessed using Kappa statistics. The CD insertion variants that are significant univariate predictors of choledocholithiasis were entered into a logistic regression model to adjust for age. Significance level was accepted at $p<0.05$ for all statistical analyses.

\section{Results}

Overall 307 (156 males, 151 females) patients (124 with choledocholithiasis and 183 controls) constituted the final study population. Out of 124 choledocholithiasis patients, $61(49.2 \%)$ were males and $63(50.8 \%)$ were females, and the median age was 66.5 (54.25-66.5) years. Out of 183 controls, 95 (51.9\%) were males and $88(48.1 \%)$ were females, and the median age was 56 (43-69) years. The gender rate $(p=0.64)$ did not differ between the groups but the controls were younger $(p<0.001)$.

In the whole cohort, the $\mathrm{CD}$ insertion was normal in $158(51.5 \%)$ and variant in 149 (48.5\%) subjects. Substantial inter-reader agreement for assessment of variation types was observed (Cohen's Kappa 0.748, $p<0.001$ ).

The craniocaudal insertion level was high in 18 (5.9\%), mid in $269(87.6 \%)$ and low in $20(6.5 \%)$ cases. The site of insertion in the transverse plane was lateral in $176(57.3 \%)$, medial in $55(17.9 \%)$, posterior in $70(22.8 \%)$, anterior in $6(2 \%)$ cases. Among the intersections of craniocaudal insertion level and transverse insertion site, the most common variation type was mid-posterior [70 (22.8\%)]. All intersections of insertion planes are demonstrated in Table 2. The normal insertion and all variant insertions of $C D$ encountered in the current study are demonstrated in Fig. 2

A total of 38 CDs had a parallel course, with 7 of them having normal mid-lateral insertion. Among the remaining 31 cases with variant insertions, the most common variant insertion pattern was low-medial [14 (36.8\%)] followed by mid-medial [10 (26.3\%)], mid-posterior [7 (18.4)].

Table 2 The distribution of all CD insertion patterns in the whole cohort

\begin{tabular}{lccccc}
\hline Whole cohort & \multicolumn{2}{c}{ Horizontal insertion site $[\boldsymbol{n}(\%)$} & Total \\
\cline { 2 - 5 } & Lateral & Medial & Posterior & Anterior & \\
\hline Craniocaudal insertion level, $n(\%)$ & & & \\
High & $18(5.9)$ & $0(0)$ & $0(0)$ & $0(0)$ & $18(5.9)$ \\
Mid & $158(51.5)$ & $35(11.4)$ & $70(22.8)$ & $6(2)$ & $267(87.6)$ \\
Low & $0(0)$ & $20(5.5)$ & $0(0)$ & $0(0)$ & $20(6.5)$ \\
Total & $176(57.4)$ & $55(16.9)$ & $70(22.8)$ & $6(2)$ & $307(100)$ \\
\hline
\end{tabular}




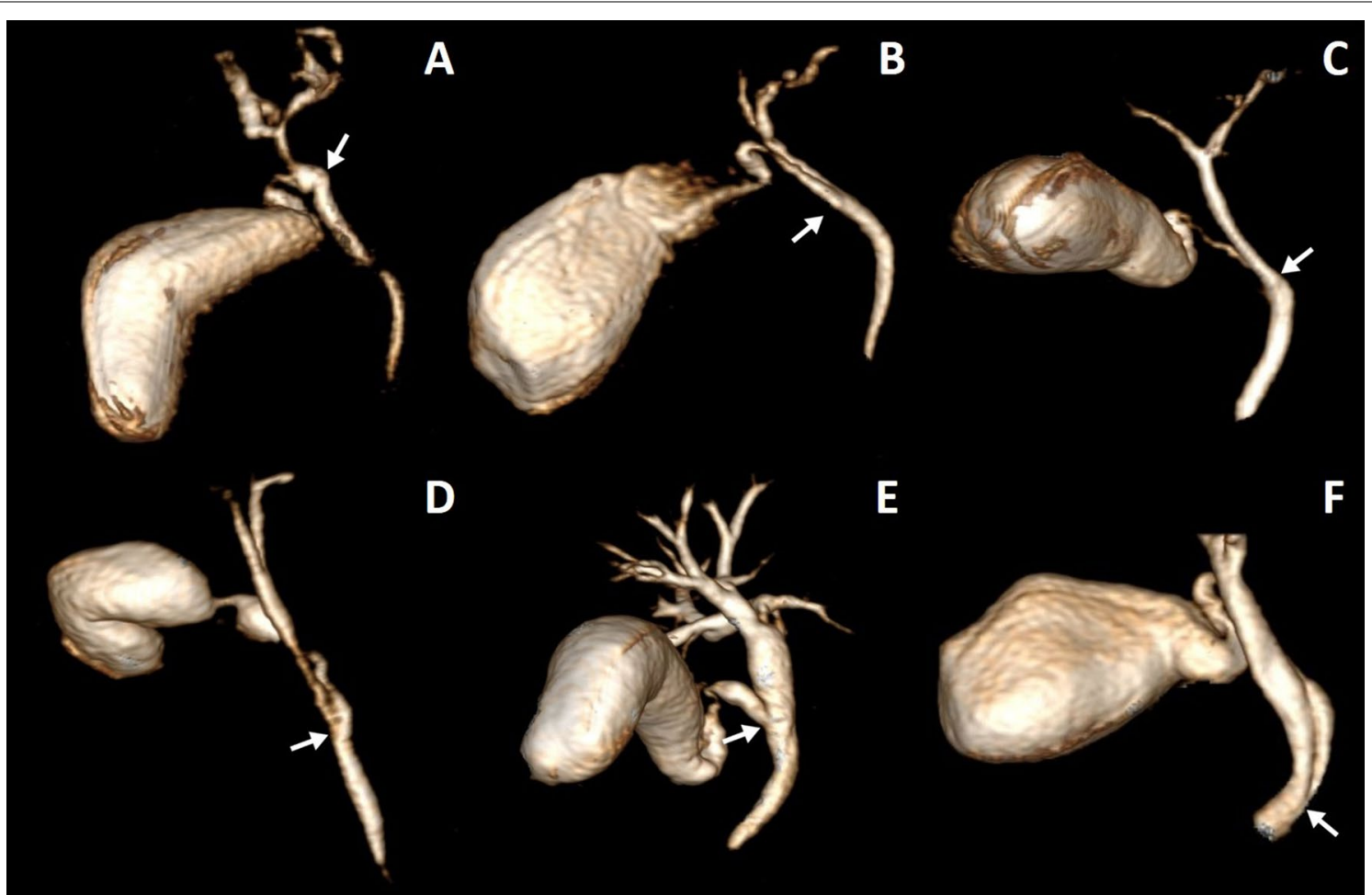

Fig. 2 The 3D volume rendering MRCP images demonstrate the high-lateral (a), mid-anterior (b), mid-posterior (c), mid-medial (d), mid-lateral (e), and low-medial (f) insertions of the cystic duct

\section{Comparison of $C D$ insertion variations}

\section{between choledocholithiasis and control groups}

The rate of total CD insertion variations was significantly lower in choledocholithiasis group than controls [50 (40.3\%) vs. 99 (54.1\%), respectively, $p=0.018$ ]. The frequencies of transverse plain insertion site were simi$\operatorname{lar}(p=0.314)$ between groups (Table 3$)$. The frequencies of craniocaudal insertion level were different between groups ( $p=0.003$ ) driven by a significantly a lower rate of low insertion [2 (1.6\%) vs. 18 (9.8\%), respectively] and a higher rate of mid insertion [118 (95.2\%) vs. 151 (82.5\%), respectively] in choledocholithiasis group (Table3).

The frequencies of each intersections of the variations in the craniocaudal and transverse plains in

Table 3 Intergroup differences of CD insertion variants

\begin{tabular}{lcc}
\hline CD insertion & Choledocholithiasis $(\boldsymbol{n}=\mathbf{1 2 4})$ & Controls $(\boldsymbol{n}=\mathbf{1 8 3})$ \\
\hline Variation $[n(\%)]$ & $50(40.3)$ & $99(54.1)$ \\
Present & $74(59.7)$ & $84(45.9)$ \\
Absent & & $\mathbf{0 . 0 1 8}$ \\
Craniocaudal insertion level $[n(\%)]$ & $4(3.2)$ & $14(7.7)$ \\
High & $118(95.2)$ & $151(82.5)$ \\
Mid & $2(1.6)$ & $18(9.8)$ \\
Low* & & \\
Horizontal insertion site $[n(\%)]$ & $78(62.9)$ & $98(53.6)$ \\
Lateral & $17(13.7)$ & $38(19.1)$ \\
Medial & $26(21)$ & $44(25.7)$ \\
Posterior & $3(2.4)$ & $3(1.6)$ \\
Anterior &
\end{tabular}

The $p$ values of comparisons with significant $(p<0.05)$ intergroup difference are represented as bold and italics

*Post hoc analysis revealed a significant difference $(p<0.05)$ for only low insertion pattern. High and mid insertions remained similar $(p>0.0 .5$ for all) between groups 
choledocholithiasis and control groups are demonstrated in Table 4. A normal lateral insertion was more common in the choledocholithiasis group (Fig. 3). The only insertion variation that significantly differed between groups was the low-medial insertion as per the post hoc analyses.

A parallel course was less frequent in the choledocholithiasis group than controls [9 (7.3\%) vs. 29 (15.8), respectively, $p=0.025$.

A binary logistic regression analysis model including the low-medial insertion, parallel course and age was constructed for prediction of choledocholithiasis. The low-medial insertion was $80.7 \%$ less likely associated with choledocholithiasis (Odds ratio: 0.193, 95\% Confidence interval: $0.039-0.954, p=0.044)$, the age (Odds ratio: 1.035, 95\% Confidence interval: 1.02-1.05, $p<0.001)$ also independently predicted choledocholithiasis, but the parallel course did not show a significant association (Odds ratio: 0.515, 95\% Confidence interval: $0.211-1.25, p=0.144)$.

Table 4 Comparison of $C D$ insertion patterns between choledocholithiasis and control groups

\begin{tabular}{lcll}
\hline $\begin{array}{l}\text { CD insertion } \\
\text { pattern }\end{array}$ & $\begin{array}{l}\text { Choledocholithiasis } \\
(\boldsymbol{n}=\mathbf{1 2 4})\end{array}$ & Controls $(\boldsymbol{n}=\mathbf{1 8 3})$ & $\boldsymbol{P}$ \\
\hline High lateral & $4(3.2)$ & $14(7.7)$ & $\mathbf{0 . 0 1 9}$ \\
Mid lateral* $^{*}$ & $\mathbf{7 4 ( 5 9 . 7 )}$ & $\mathbf{8 4}(\mathbf{4 5 . 9 )}$ & \\
Mid medial & $15(12.1)$ & $20(10.9)$ & \\
Mid posterior & $26(21)$ & $44(24)$ & \\
Mid anterior & $3(2.4)$ & $3(1.6)$ & \\
Low medial $^{*}$ & $\mathbf{2 ( 1 . 6 )}$ & $\mathbf{1 8 ( 9 . 8 )}$ & \\
\hline
\end{tabular}

The $p$ values of comparisons with significant $(\mathrm{p}<0.05)$ intergroup difference are represented as bold and italics

*Post hoc tests revealed significant difference between groups only for midlateral and low-medial insertions

\section{Discussion}

The main findings in the current study were: (1) The prevalence of $C D$ insertion variations in an unselected population undergoing MRCP is quite high (49.2\%); (2) Mid-posterior insertion is the most common (22.8\%) variant type; (3) A CD with high insertion joins exclusively through the lateral aspect of extrahepatic bile duct; (4) A CD with low insertion joins exclusively through medial aspect of extrahepatic bile duct; (5) A variant insertion is less frequent in patients with choledocholithiasis than controls; (6) A low-medial insertion independently predicts the absence of choledocholithiasis (Fig. 4).

The relatively high rate of $C D$ insertion variations in current study is close to that observed in a previous report which included an unselected MRCP population [2]. The prevalence of $\mathrm{CD}$ anatomical variations has been reported between 8.3 and $24 \%$ in surgical series [10-12] which is lesser than the rate observed in current or previous MRCP cohorts.

Three most common variations of cystic duct have been previously described as low insertion, medial insertion and insertion after a parallel course in earlier studies [13, 14]. More recent studies have reported that the posterior insertion was the most common anomaly of the cystic duct $[2,15]$. A mid-posterior insertion was also the most frequent [(70 (22.8\%)] variation type in the current study followed by mid-medial insertion. For this study, a parallel course $(n=34)$ was not considered a CD insertion anomaly, yet $20 \%$ of them showed normal mid-lateral insertion. Since a more than $2-\mathrm{cm}$ long proximal-to-distal extension is required for a parallel course, it is not surprising that none of the CDs with a parallel course had a high insertion. All showed either a mid or low insertions.

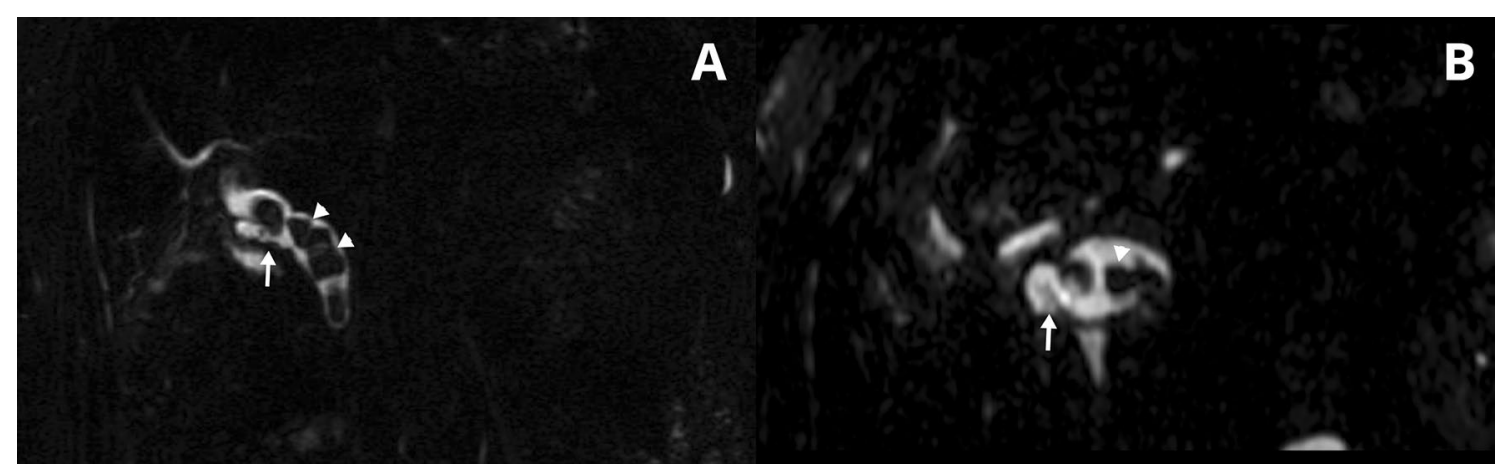

Fig. 3 A patient with mid-lateral insertion of cystic duct and choledocholithiasis. The coronal a MRCP image depicts the mid insertion level, and the axial $\mathbf{b}$ MRCP image shows the lateral insertion site. Note the insertion point (arrows) and the multiple stones (arrowheads) in the common bile duct 


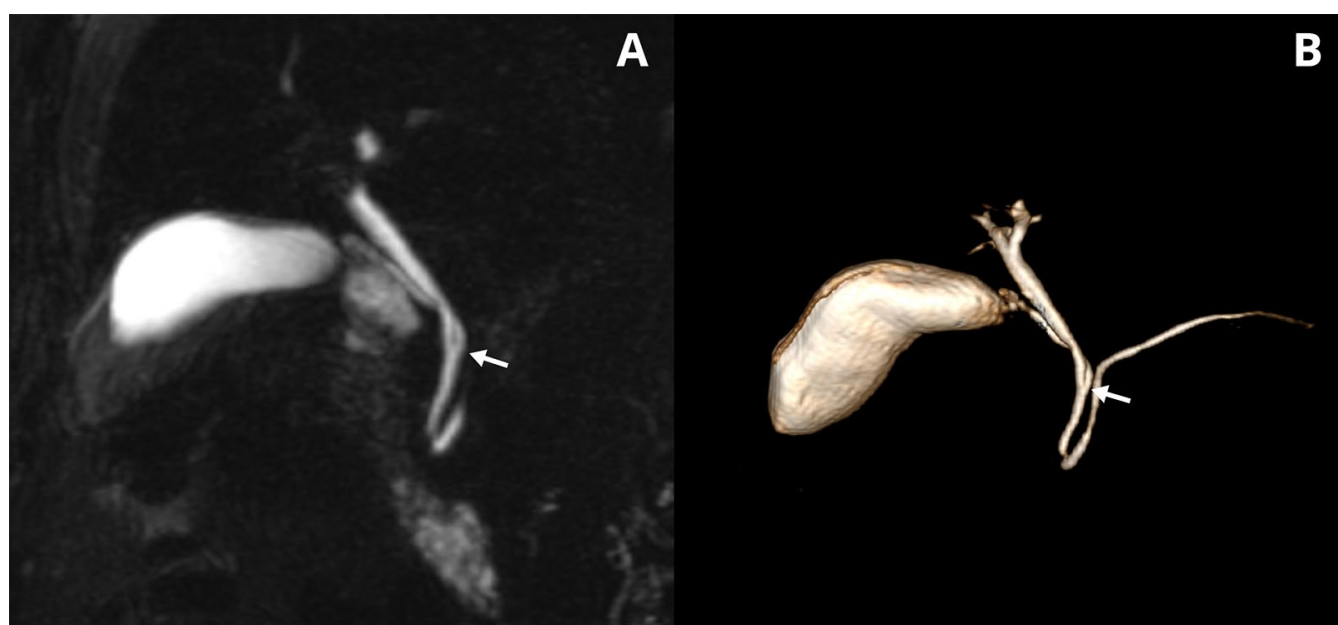

Fig. 4 A patient with low-medial insertion of cystic duct with no choledocholithiasis. The Coronal (a) and 3D volume rendering (b) MRCP images demonstrate the low medial insertion (white arrow) of cystic duct with no stone in the common bile duct

Of note, CDs with a high insertion were exclusively lateral, and those with a low insertion were exclusively medial in the current study.

A recent study has found an association between intrapancreatic low insertion cystic duct and choledocholithiasis [16]. However, a plausible underlying mechanism for how low insertion may trigger stone formation in the common bile duct was not provided. A low insertion has been associated with biliary stones in previous studies. The stagnation of biliary flow in CD's with low-insertion has been proposed that would produce the formation of biliary stone. However, this hypothesis is not evidence based. Moreover, expecting stagnation of bile flow in CD's would be more plausible in those with long parallel or spiral course variations, rather than in those with insertion variations. Indeed, a low medial insertion was less commonly associated with choledocholithiasis even after adjustment for age and presence of parallel course, in the current work. This observation is contradictory to previous reports, and may be explained by the differences between populations studied in terms of biochemical and clinical contributors to stone formation. However, investigating all of the biological triggers of gallstone formation was beyond the scope of our study and was not evaluated. Although the genesis of gallstone due to stagnation of bile in patients with variant insertion of $C D$ is common sense, this does not automatically mean that the stone will subsequently travel to common bile duct. We argue that, the reduced flow may even slow or preclude the movement of the gallstone through the $\mathrm{CD}$, which may explain the relatively lower frequency of choledocholithiasis in patients with variant CD insertions in the current study.
The main limitation of our study is that our results are derived from an unselected MRCP population with or without choledocholithiasis and may not be extrapolated to the general population. The lack of surgical confirmation of $C D$ insertions may be considered another limitation.

The strengths of our study are the relatively large sample size, presence of control group and standardized definitions of $C D$ insertion variations. The $C D$ variations including the size or course were not considered as insertion variations and the relative frequencies in our study reflect the exact insertion patterns.

\section{Conclusions}

The prevalence of CD insertion variations in an unselected MRCP population is quite high and a mid-posterior insertion is the most common variant type. The CDs with high insertion joins exclusively through the lateral aspect and those with low insertion joins exclusively through the medial aspect of extrahepatic bile duct. A low-medial insertion independently predicts the absence of choledocholithiasis.

\section{Abbreviations}

CD: cystic duct; CBD: common bile duct; MRCP: magnetic resonance cholangiopancreatography.

\section{Acknowledgements}

No acknowledgements: not applicable.

\section{Authors' contributions}

NG (corresponding author) was responsible for project development and final approval of the manuscript. NG (corresponding author), MBD, MA and MY collected the data. NG (corresponding author) and UPOS were involved in manuscript writing. NG (corresponding author), UPOS and BA were involved 
in critical review. BA was involved in manuscript editing. MBD analyzed the data. All authors read and approved the final manuscript.

\section{Funding}

None; not applicable.

\section{Availability of data and materials}

The datasets used and/or analyzed during the current study are available from the corresponding author on reasonable request.

\section{Declarations}

Ethics approval and consent to participate

“Istanbul Medeniyet University Goztepe Prof. Dr. Suleyman Yalcin City Hospital Ethics Committee" approval was obtained. Decision Number: 2020/0068.

\section{Consent for publication}

No clinical photographs; not applicable.

\section{Competing interests}

The author(s) declared no potential conflicts of interest with respect to the research, authorship, and/or publication of this article.

\section{Author details}

${ }^{1}$ Department of Radiology, Göztepe Pof. Dr. Süleyman Yalçın City Hospital, Faculty of Medicine, İstanbul Medeniyet University, Eğitim M, Dr. Erkin C. No: 161/1, 34722 Kadıköy, İstanbul, Turkey. ${ }^{2}$ Department of Radiology, Göztepe Pof. Dr. Süleyman Yalçın City Hospital, Eğitim M, 34722 Kadıköy, İstanbul, Turkey.

Received: 17 May 2021 Accepted: 2 Auqust 2021

Published online: 13 August 2021

\section{References}

1. Doherty GM, Way LW (2006) Biliary tract. In: Doherty GM (ed) Current surgical diagnosis and treatment, 12th edn. McGraw Hill Companies, New York, pp 576-601

2. Sarawagi R, Sundar S, Gupta SK et al (2016) Anatomical variations of cystic ducts in magnetic resonance cholangiopancreatography and clinical implications. Radiol Res Pract 2016:3021484. https://doi.org/10.1155/ 2016/3021484

3. Onder H, Ozdemir MS, Tekbaş G, Ekici F, Gümüş H, Bilici A (2013) 3-T MRI of the biliary tract variations. Surg Radiol Anat 35(2):161-167. https://doi. org/10.1007/s00276-012-1021-0

4. Aljiffry M, Abbas M, Wazzan MAM, Abduljabbar AH, Aloufi S, Aljahdli E (2020) Biliary anatomy and pancreatic duct variations: a cross-sectional study. Saudi J Gastroenterol 26(4):188-193. https://doi.org/10.4103/sjg. SJG $573 \quad 19$
5. Taştemur Y (2020) Anatomical variations of the cystic duct in Turkish population and their association with biliary track stone. J Coll Physicians Surg Pak 30(10):1005-1008. https://doi.org/10.29271/jcpsp.2020.10.1005

6. Tsitouridis I, Lazaraki G, Papastergiou C et al (2007) Low conjunction of the cystic duct with the common bile duct: does it correlate with the formation of common bile duct stones? Surg Endosc 21(1):48-52. https:// doi.org/10.1007/s00464-005-0498-6

7. Kao JT, Kuo CM, Chiu YC et al (2011) Congenital anomaly of low insertion of cystic duct: endoscopic retrograde cholangiopancreatography findings and clinical significance. J Clin Gastroenterol 45(7):626-629. https:// doi.org/10.1097/MCG.0b013e31821bf824

8. Sirinek KR, Schwesinger WH (2015) Has intraoperative cholangiography during laparoscopic cholecystectomy become obsolete in the era of preoperative endoscopic retrograde and magnetic resonance cholangiopancreatography? J Am Coll Surg 220(4):522-528. https://doi.org/10. 1016/j.jamcollsurg.2014.12.043

9. Kang KA, Kwon HJ, Ham SY et al (2020) Impacts on outcomes and management of preoperative magnetic resonance cholangiopancreatography in patients scheduled for laparoscopic cholecystectomy: for whom it should be considered? Ann Surg Treat Res 99(4):221-229. https://doi.org/ 10.4174/astr.2020.99.4.221

10. Puente SG, Bannura GC (1983) Radiological anatomy of the biliary tract: variations and congenital abnormalities. World J Surg 7(2):271-276. https://doi.org/10.1007/BF01656159

11. Khan AS, Paracha SA, Shah Z et al (2012) Anatomical variations of cystic duct encountered during open cholecystectomy. Khyber Med Univ J 4(1):19-22

12. Talpur KA, Laghari AA, Yousfani SA et al (2010) Anatomical variations and congenital anomalies of extra hepatic biliary system encountered during laparoscopic cholecystectomy. J Pak Med Assoc 60(2):89-93

13. Mortelé KJ, Ros PR (2001) Anatomic variants of the biliary tree: MR cholangiographic findings and clinical applications. AJR Am J Roentgenol 177(2):389-394. https://doi.org/10.2214/ajr.177.2.1770389

14. Taourel P, Bret PM, Reinhold C et al (1996) Anatomic variants of the biliary tree: diagnosis with MR cholangiopancreatography. Radiology 199:521-527

15. Swain B, Sahoo RK, Sen KK, Manoj K, Parihar SS, Dubey R (2020) Evaluation of intrahepatic and extrahepatic biliary tree anatomy and its variation by magnetic resonance cholangiopancreatography in Odisha population: a retrospective study. Anat Cell Biol 53(1):8-14. https://doi.org/10.5115/acb. 19.177

16. Renzulli M, Brocchi S, Marasco G, Spinelli D, Balacchi C, Barakat M, Pettinari I, Golfieri R (2020) A new quantitative classification of the extrahepatic biliary tract related to cystic duct implantation. J Gastrointest Surg. https://doi.org/10.1007/s11605-020-04852-8

\section{Publisher's Note}

Springer Nature remains neutral with regard to jurisdictional claims in published maps and institutional affiliations.

\section{Submit your manuscript to a SpringerOpen ${ }^{\odot}$ journal and benefit from:}

- Convenient online submission

- Rigorous peer review

- Open access: articles freely available online

- High visibility within the field

- Retaining the copyright to your article

Submit your next manuscript at springeropen.com 